\title{
5G NR system design: a concise survey of key features and capabilities
}

\author{
Federica Rinaldi ${ }^{1}$ (I) $\cdot$ Alessandro Raschellà ${ }^{2} \cdot$ Sara Pizzi $^{1}$
}

Accepted: 27 September 2021 / Published online: 20 October 2021

(C) The Author(s) 2021

\begin{abstract}
As we enter a new era of next-generation wireless systems represented by Fifth Generation (5G) New Radio (NR) technology, it is essential to grasp the recent progress in their standardization and development. This article offers a concise survey of the 5G NR system design that aims at introducing its features according to the relevant Third Generation Partnership (3GPP) specifications. Our focus is set on the flexibility of 5G NR, which refers to its capability to support novel services and technologies, such as enhanced Mobile Broadband (eMBB) and Internet of Things (IoT) for massive Machine Type Communications (mMTC) while satisfying the underlying quality requirements. The key enablers of the 5G NR operation are scalable numerology, ultra-lean and beam-centric design, support for low latency, spectrum extension, and forward compatibility. This work summarizes these important features by studying the overall $5 \mathrm{G}$ architecture and the user-/control-plane protocol stacks specified by 3GPP. Furthermore, the impact of scalable numerology on system performance is discussed. Finally, we also consider open challenges and future research directions.
\end{abstract}

Keywords $5 \mathrm{G} \cdot$ New radio $\cdot$ Scalable numerology $\cdot 5 \mathrm{G}$ use cases

$\begin{array}{ll}\text { Abbreviations } & \\ \text { 3GPP } & \text { Third Generation Partnership } \\ \text { 5G } & \text { Fifth Generation } \\ \text { 5GC } & \text { 5G Core Network } \\ \text { 5G-EIR } & \text { 5G-Equipment Identity Register } \\ \text { AF } & \text { Application Function } \\ \text { AM } & \text { Acknowledged Mode } \\ \text { AMF } & \text { Access and Mobility Management } \\ & \text { Function } \\ \text { AS } & \text { Access Stratum } \\ \text { AUSF } & \text { Authentication Server Function } \\ \text { BCCH } & \text { Broadcast Control Channel } \\ \text { BCH } & \text { Broadcast Channel } \\ \text { BSR } & \text { Buffer Status Reporting } \\ \text { BWP } & \text { Bandwidth Part }\end{array}$

Federica Rinaldi

federica.rinaldi@unirc.it

Alessandro Raschellà

a.raschella@ljmu.ac.uk

Sara Pizzi

sara.pizzi@unirc.it

1 DIIES Department, University Mediterranea of Reggio Calabria, Reggio Calabria, Italy

2 School of Computer Science and Mathematics, Liverpool John Moores University (LJMU), Liverpool, UK

$\begin{array}{ll}\text { CCCH } & \text { Common Control Channel } \\ \text { CDSA } & \text { Control/Data Separation Architecture } \\ \text { CHF } & \text { CHarging Function } \\ \text { CN } & \text { Core Network } \\ \text { CORESET } & \text { Control Resource Set } \\ \text { CP } & \text { Control Plane } \\ \text { CPF } & \text { Control Plane Function } \\ \text { CP-OFDM } & \text { Orthogonal Frequency Division Multi- } \\ & \text { plexing with Cyclic Prefix } \\ \text { CQI } & \text { Channel Quality Indicator } \\ \text { CRI } & \text { CSI-RS Resource Indicator } \\ \text { CSI } & \text { Channel State Information } \\ \text { CSI-RS } & \text { Channel State Information Reference } \\ & \text { Signal } \\ \text { CU } & \text { Central Unit } \\ \text { DCCH } & \text { Dedicated Control Channel } \\ \text { DFT-s-OFDM } & \text { Discrete Fourier Transform-spread- } \\ & \text { OFDM } \\ \text { DMRS } & \text { Demodulation Reference Signal } \\ \text { DL-SCH } & \text { Downlink Shared Channel } \\ \text { DRX } & \text { Discontinuous Reception } \\ \text { DTCH } & \text { Dedicated Traffic Channel } \\ \text { DU } & \text { Distributed Unit } \\ \text { eMBB } & \text { enhanced Mobile Broadband } \\ \text { EN-DC } & \text { E-UTRAN NR-Dual Connectivity } \\ \text { EPC } & \text { Evolved Packet Core } \\ & \end{array}$




\begin{tabular}{|c|c|c|c|}
\hline E-UTRA & $\begin{array}{l}\text { Evolved Universal } \\
\text { Access }\end{array}$ & $\begin{array}{l}\text { PDU } \\
\text { PEI }\end{array}$ & $\begin{array}{l}\text { Packet Data Unit } \\
\text { Permanent Equipment Identifier }\end{array}$ \\
\hline E-UTRAN & $\begin{array}{l}\text { Evolved Universal Terrestrial Radio } \\
\text { Access Network }\end{array}$ & $\begin{array}{l}\text { PHY } \\
\text { PRACH }\end{array}$ & $\begin{array}{l}\text { Physical } \\
\text { Physical Random Access Channel }\end{array}$ \\
\hline FDD & Frequency Division Duplex & PRB & Physical Resource Block \\
\hline FR & Frequency Range & PRS & Positioning Reference Signal \\
\hline gNB & 5G NodeB & PSS & Primary Synchronization Signal \\
\hline HTC & Holographic Type Communications & PTRS & Phase-Tracking Reference Signal \\
\hline ICI & Inter-Carrier Interference & PUCCH & Physical Uplink Control Channel \\
\hline IoT & Internet of Things & PUSCH & Physical Uplink Shared Channel \\
\hline IRS & Intelligent Reflecting Surfaces & QFI & QoS Flow Identifier \\
\hline ITU & International Telecommunications Union & QoS & Quality of Service \\
\hline KPI & Key Performance Indicator & $\mathrm{RACH}$ & Random Access Channel \\
\hline LCG & Logical Channel Group & RAN & Radio Access Network \\
\hline LCP & Logical Channel Prioritization & RAT & Radio Access Technology \\
\hline LI & Layer Indicator & RI & Rank Indicator \\
\hline LTE & Long Term Evolution & RLC & Radio Link Control \\
\hline MAC & Medium Access Control & $\mathrm{RRC}$ & Radio Resource Control \\
\hline MCG & Master Cell Group & RRM & Radio Resource Management \\
\hline MCS & Modulation and Coding Scheme & RS & Reference Signal \\
\hline MIB & Master Information Block & SCG & Secondary Cell Group \\
\hline MIMO & Multiple-Input Multiple-Output & SCS & Subcarrier spacing \\
\hline mMTC & massive Machine Type Communications & SDAP & Service Data Adaptation Protocol \\
\hline MN & Master Node & SDU & Service Data Unit \\
\hline MR-DC & Multi-Radio Dual Connectivity & SMF & Session Management Function \\
\hline MU-MIMO & Multi-User Multiple-Input Multiple- & SN & Secondary Node \\
\hline & Output & SR & Scheduling Request \\
\hline mmWave & millimeter-Wave & SRB & Signaling Radio Bearers \\
\hline NAS & Non-Access Stratum & SRS & Sounding Reference Signal \\
\hline NE-DC & NR E-UTRAN-Dual Connectivity & SSBRI & SS/PBCH Block Resource Indicator \\
\hline NEF & Network Exposure Function & SSS & Secondary Synchronization Signal \\
\hline NG & Next Generation & SUL & Supplementary Uplink \\
\hline ng-eNB & new generation LTE NodeB & TDD & Time Division Duplex \\
\hline NGEN-DC & Next-Generation-Dual Connectivity & $\mathrm{TM}$ & Transparent Mode \\
\hline NG-RAN & Next-Generation Radio Access Network & TTI & Transmission Time Interval \\
\hline NOMA & Non-Orthogonal Multiple Access & UCMF & UE radio Capability Management \\
\hline NR & New Radio & & Function \\
\hline NR-DC & NR-NR Dual Connectivity & UDM & Unified Data Management \\
\hline NRF & Network Repository Function & UDR & Unified Data Repository \\
\hline NSSAAF & $\begin{array}{l}\text { Network Slice Specific Authentication } \\
\text { and Authorization Function }\end{array}$ & $\begin{array}{l}\text { UDSF } \\
\text { UE }\end{array}$ & $\begin{array}{l}\text { Unstructured Data Storage Function } \\
\text { User Equipment }\end{array}$ \\
\hline NSSF & Network Slice Selection Function & UL-SCH & Uplink Shared Channel \\
\hline NTN & Non-Terrestrial Network & UM & Unacknowledged Mode \\
\hline NWDAF & Network Data Analytics Function & UP & User Plane \\
\hline OFDM & $\begin{array}{ll}\text { Orthogonal } & \text { Frequency-Division } \\
\text { Multiplexing } & \end{array}$ & $\begin{array}{l}\text { UPF } \\
\text { uRLLC }\end{array}$ & $\begin{array}{l}\text { User Plane Function } \\
\text { ultra-Reliable Low }\end{array}$ \\
\hline PBCH & Physical Broadcast Channel & & Communications \\
\hline $\mathrm{PCCH}$ & Paging Control Channel & & \\
\hline $\mathrm{PCH}$ & Paging Channel & & \\
\hline PCF & Policy Control Function & & \\
\hline $\mathrm{PDCCH}$ & Physical Downlink Control Channel & & \\
\hline PDCP & Packet Data Convergence Protocol & & \\
\hline PDSCH & Physical Downlink Shared Channel & & \\
\hline
\end{tabular}




\section{Introduction}

The Fifth Generation (5G) wireless system, whose air interface is known as "New Radio" (NR) [1], represents the most promising next-generation solution to meet the needs of the increasingly demanding mobile market. The $5 \mathrm{G}$ NR provides dissimilar types of service characterized by different requirements.

In this regard, the International Telecommunication Union (ITU) [2] defines the macro-categories of use cases: (i) enhanced Mobile Broadband (eMBB) for services with high data-rates; (ii) machine-type devices interconnected through the Internet of Things (IoT) for massive Machine Type Communications (mMTC) that require low cost, low power consumption, and long battery life; to connect numerous devices with low cost, low power consumption, and long battery life; and (iii) Ultra-Reliable Low Latency Communications (URLLC) for low latency two-way transfers between devices under extreme network reliability.

For 5G NR standardization, the Third Generation Partnership (3GPP) reconsiders many aspects of a wireless system, e.g., the Key Performance Indicator (KPI) targets, network architecture, radio access network (RAN) functions, and the entire network protocol stack. As a result, 5G NR [3] can provide multiple benefits as compared to Long Term Evolution (LTE).

First, an extension of the spectrum range allows NR to support operations in licensed bands from below $1 \mathrm{GHz}$ to $52.6 \mathrm{GHz}$. This is different from LTE, which operates in the licensed spectrum at $3.5 \mathrm{GHz}$ and unlicensed spectrum at $5 \mathrm{GHz}$. At millimeter-wave (mm-Wave) frequencies, high capacity and extreme data rates are possible, even though higher frequencies introduce limitations in coverage due to increased signal attenuation. Further, scalable numerology [4] represents a major 5G innovation to offer network flexibility. Indeed, NR may adjust its subcarrier spacing (SCS), which is fixed to $15 \mathrm{kHz}$ in LTE [5].

Second, 5G NR is designed ultra-lean to lower interference and increase energy efficiency by reducing alwayson transmissions, which is a crucial aspect to extend the lifetime of IoT devices. 5G NR is beam-centric by extending beamforming and multi-antenna schemes from data transmission to control-plane procedures and initial access. 5G NR also ensures forward compatibility as it is prepared for its future evolution in use cases and technologies. Here, the introduction of mini-slots makes $5 \mathrm{G} \mathrm{NR}$ able to guarantee low-latency requirements (e.g., for URLLC).

Finally, NR extends the LTE concept of carrier aggregation by supporting the so-called Supplementary Uplink (SUL). In contrast to carrier aggregation wherein each uplink carrier is associated with a certain downlink carrier, in the case of SUL, a conventional downlink/uplink carrier is associated with a supplementary uplink carrier operating at lower frequencies. The objective of SUL is to extend uplink coverage and increase uplink data rates in the case of limited power owing to reduced path loss in low-frequency bands.

In this article, we first concisely review the 5G NR system by focusing on the $5 \mathrm{G}$ network architecture design and user-/control-plane protocol stacks specified by 3GPP in the 38th specification series. Then, we analyze the $5 \mathrm{G}$ NR system performance under varying channel bandwidths for all numerologies. Our goal is to offer the reader-as a one-stop tutorial that is clear and accessible - an overview of the key $5 \mathrm{G}$ NR concepts.

In the literature, many works deal with the $5 \mathrm{G}$ NR technology by focusing on the main research problems, e.g., mobility management [6], energy efficiency [7], beamforming and antenna configuration [8], and radio resource management (RRM). In particular, RRM is an extremely important research topic for $5 \mathrm{G}$ NR systems, including both terrestrial and non-terrestrial networks (NTNs). Regarding this latter, in [9], an innovative RRM approach has been proposed for 5G NR multi-beam NTNs. Furthermore, the dynamic spectrum management has been addressed in [10] for simultaneous transmissions via licensed and unlicensed bands in $5 \mathrm{G}$ wireless networks based on Non-Orthogonal Multiple Access (NOMA), which was initially considered to be a 3GPP study item for $5 \mathrm{G}$ NR but then it was left for beyond 5G [11]. Other works overview the main features of the 5G NR technology. They are listed in Table 1 where we also summarize our contributions.

The remainder of the paper is organized as follows. In Sect. 2, the overall $5 \mathrm{G}$ architecture is presented. Then, the $5 \mathrm{G}$ NR physical layer is summed up in Sect. 3. Sections 4 and 5 describe the $5 \mathrm{G}$ radio protocol architecture for userplane and control-plane, respectively. The 5G NR system performance is evaluated under different numerologies in Sect. 6. Open challenges and future research directions are illustrated in Sect. 7. Finally, conclusions are drawn in Sect. 8.

\section{The 5G system architecture}

The 5G NR architecture comprises next-generation RAN (NG-RAN) and 5G Core Network (5GC) [15], both described in the following subsections. 
Table 1 Related works on 5G NR

Year Publication Brief description

2016 [4] The paper proposes a flexible physical-layer design based on OFDM with scalable numerology for all link types to satisfy the $5 \mathrm{G}$ requirements and support various carrier frequencies and deployments.

2017 [1] The paper investigates the key features of the 5G NR air interface, such as waveforms, multiple access techniques, forward compatibility, and advanced technologies to improve performance and efficiency.

2017 [3] The paper provides an overview of the key NR technology components including flexible numerology and frame structure, massive MIMO, interworking between high and low frequencies, and ultra-lean communications.

2017 [12] The review focuses on the NR physical layer components: modulation schemes and channel coding, waveforms and frame structure, reference signals, and multi-antenna transmissions.

2018 [6] The paper investigates multi-beam operation in NR systems by focusing on initial access and random access procedures, system information, and synchronization mechanisms.

2018 [13] The paper overviews the 5G physical-layer technology by describing the fundamental NR concepts, such as waveform, numerology, frame structure, modulation and coding, physical channels, and reference signals.

2018 [14] The paper overviews the 5G NR air interface improvements introduced by 3GPP Release 15 and focuses on the directional access in mmWave-based NR systems, whose performance has been evaluated analytically and through simulations.

Our

This article surveys NR by focusing on: the overall architecture of 5G NR and dual connectivity architecture options;

contributions innovations introduced by the NR technology in the user-/control-plane protocol stacks; first-order simulation-based analysis regarding the impact of scalable numerology on 5G NR performance; future research directions by $3 \mathrm{GPP}$.

\subsection{The NG-RAN}

The NG-RAN includes new generation LTE eNodeB (ngeNB) and 5G NodeB (gNB), which are responsible for the radio functions, e.g., RRM, admission and connection control, and Quality of Service (QoS) flow management. The ng-eNB employs Evolved-Universal Terrestrial Radio Access (E-UTRA) user-/control- plane protocols to serve LTE User Equipements (UEs) and is connected to the $5 \mathrm{GC}$ via the NG interface.

Figure 1 depicts the NG-RAN architecture consisting of a set of gNBs [16]. The gNB employs NR user-/controlplane protocols to serve NR UEs and is connected to the $5 \mathrm{GC}$ via the NG interface and to other gNBs through the $\mathrm{Xn}$ interface.

The gNB consists of a central unit (i.e., gNB-CU) and one or more distributed units (i.e., gNB-DU). One gNB-DU is connected to only one gNB-CU via F1 interface. NG, $\mathrm{Xn}$, and $\mathrm{F} 1$ are logical interfaces. The $\mathrm{Xn}-\mathrm{C}$ interface

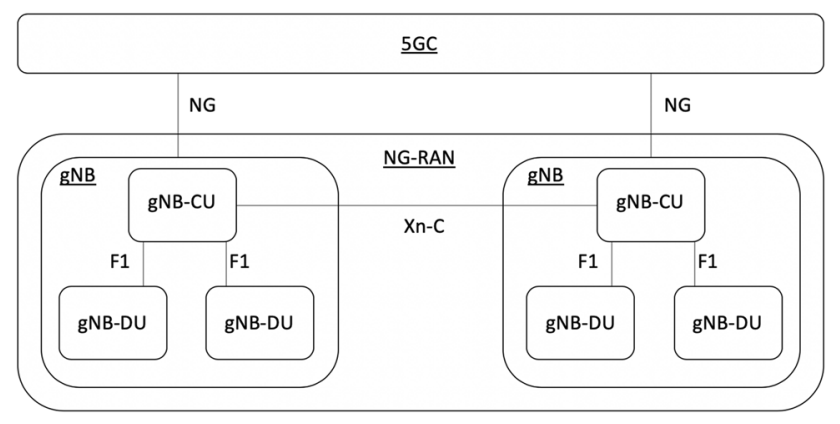

Fig. 1 NG-RAN interconnects gNB-CUs of different gNBs. The gNB can also consist of a gNB-CU control-plane (gNB-CU-CP), multiple gNB-CU user-plane (gNB-CU-UPs), and multiple gNB-DUs.

Figure 2 illustrates the overall architecture with separation of the control-plane and the user-plane for the gNB$\mathrm{CU}$ (i.e., gNB-CU-CP and gNB-CU-UP) [16]. The gNB$\mathrm{CU}-\mathrm{CP}$ is connected to the gNB-DU through the F1-C interface. A gNB-CU-UP is connected to the gNB-DU through the F1-U interface and to only one gNB-CU-CP through the E1 interface. The gNB-CU-UP is connected to only one gNB-CU-CP, to multiple gNB-CU-UPs and multiple gNB-DUs under the control of the same gNB-CUCP.

The NG-RAN offers new functions, such as: (i) network slicing, (ii) contacting UEs in inactive mode, (iii) handover between E-UTRA and NR via a direct interface between eNB and gNB, (iv) handover between E-UTRA and NR via core network (CN), (v) session management, and (vi) tight

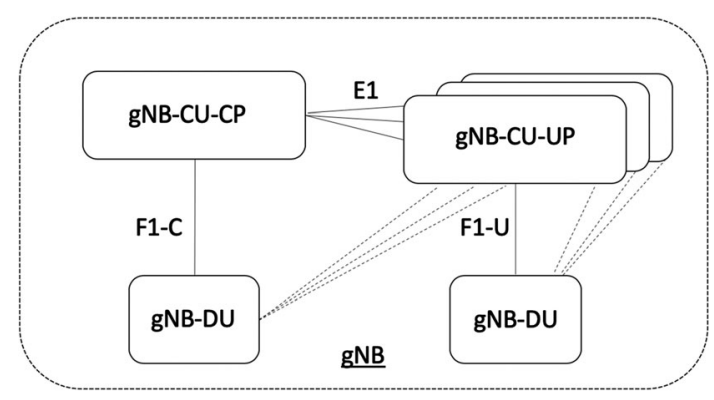

Fig. 2 gNB architecture with separation of gNB-CU-CP and gNBCU-UP 
interworking between NR and E-UTRA, and dual connectivity [17]. In Sect. 2.3, we offer further details regarding dual connectivity.

\subsection{The 5G core network}

Figure 3 shows the high-level representation of the 5GC. Detailed descriptions (i.e., reference architecture type, reference points, and point-to-point interactions between the functions) are provided in the specification [18].

The 5GC follows a service-based architecture, supports network slicing, and splits the user-plane and the controlplane. The User Plane Function (UPF) acts as a gateway to connect the RAN to external networks. In certain cases, it also represents the anchor point for intra-/inter-Radio Access Technology (RAT) mobility. UPF is responsible for packet routing, forwarding, and inspection, handling QoS, and managing traffic measurements.

Control Plane Functions (CPF) are as follows. The Session Management Function (SMF) handles session management and establishment, allocates IP addresses to the UEs, facilitates roaming, and controls the UPF. The Access and Mobility Management Function (AMF) manages registration, reachability, mobility, connection, and location services. AMF also handles access authentication and authorization, and facilitates idle-state mobility. The Non-Access Stratum (NAS) operates in-between the AMF and the device, while the Access Stratum (AS) operates inbetween the device and the RAN.

Other types of functions and entities are: Unified Data Management (UDM) that authenticates and authorizes access, Policy Control Function (PCF) providing policy rules, Authentication Server Function (AUSF) handling authentication, Application Function (AF) influencing the traffic routing, Network Exposure Function (NEF) providing secure information from external application to 3GPP network, Network Repository Function (NRF) supporting service discovery function, Unified Data Repository (UDR) responsible of storage and retrieval of subscription data by UDM, Unstructured Data Storage Function (UDSF) responsible of storage and retrieval of unstructured data by any network function, Network Data Analytics Function (NWDAF) managing network analytics, Network Slice Specific Authentication and Authorization Function (NSSAAF) and Network Slice Selection Function (NSSF) in charge of Network Slicing, UE radio Capability Management Function (UCMF) storing all UE Radio Capability ID, 5G-Equipment Identity Register (5G-EIR) checking the status of Permanent Equipment Identifier (PEI) (e.g., whether it has been blacklisted), and CHarging Function (CHF) that manages charging information. Details of these functions can be found in [18].

\subsection{LTE/NR dual connectivity}

Dual connectivity has been defined in LTE Release 12 [19] as the operation wherein at least two different eNBs, one master node $(\mathrm{MN})$ connected to a secondary node $(\mathrm{SN})$, offer radio resources to a certain UE. Radio resource aggregation improves per-user throughput and mobility robustness since UEs may be scheduled via multiple eNBs. Different from LTE, NR dual connectivity operates for nodes belonging to two different RATs, that is, the gNB and the eNB provide NR and E-UTRA/NR access, respectively. This results in tight interworking between the two radio technologies and allows NR to gradually fit into the existing LTE networks. In fact, NR access network may operate in two modes: non-standalone and standalone. Non-standalone operation offers the possibility to connect NR in the existing LTE networks, thus speeding up the $5 \mathrm{G}$ roll-out. On the other hand, standalone operation expects the connection of NR to the 5GC as well as that of LTE to the 5GC. Further details are provided in the next subsection.

\subsection{LTE-NR architecture options}

In Release 15 [17], 3GPP standardized a combined architecture design. Table 2 demonstrates different options for the two core networks, i.e., 5GC and Enhanced Packet

Fig. 3 High-level representation of the 5G Core Network

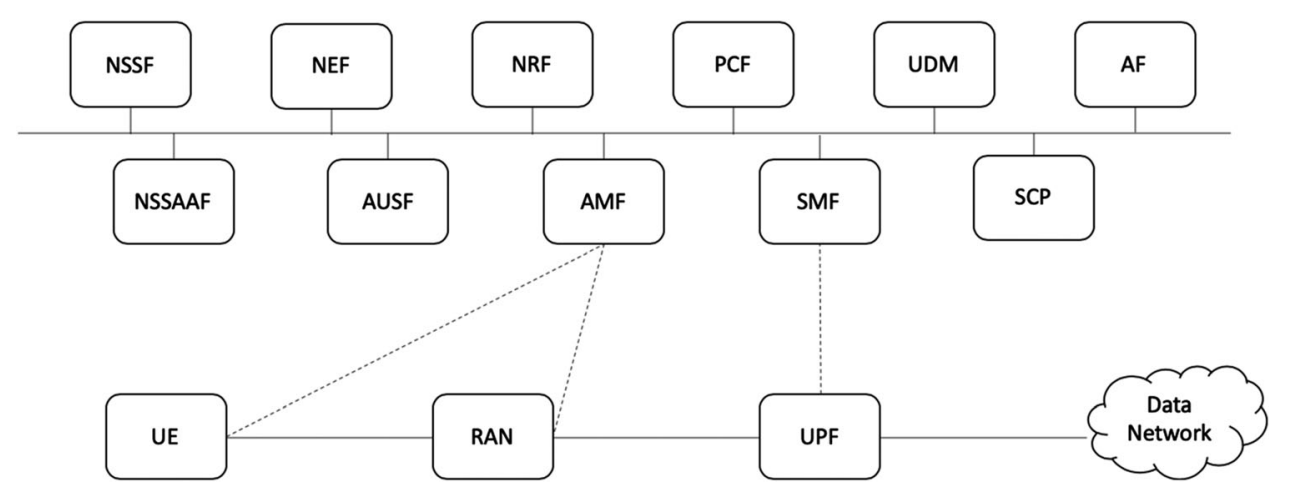


Table 2 LTE-NR combined architecture design

\begin{tabular}{lllll}
\hline DC architecture & Core network & MN & SN & Terminology \\
\hline Option 1 & EPC & eNB & - & LTE/EPC \\
Option 2 & $5 G C$ & gNB & - & NR/5GC \\
Option 3 & EPC & eNB & gNB & EN-DC \\
Option 4 & $5 G C$ & gNB & eNB & NE-DC \\
Option 5 & $5 G C$ & eNB & - & eLTE \\
Option 7 & $5 G C$ & eNB & gNB & NGEN-DC
\end{tabular}

Core (EPC), and the two RATs, i.e., NG-RAN and Evolved Universal Terrestrial Radio Access Network (E-UTRAN).

Option 1 represents the "legacy" LTE/EPC operation, where the eNB is directly linked to the LTE core network. In option 2, the gNB is directly linked to the $5 \mathrm{GC}$ in the standalone mode. In non-standalone option 3, the NG-RAN supports E-UTRAN-NR Dual Connectivity (EN-DC), where UE is connected to one eNB (MN) and one gNB (SN). The eNB is connected to the EPC via the $\mathrm{S} 1$ interface and to the gNB via the $\mathrm{X} 2$ interface. The gNB can also be connected to the EPC via the S1-U interface and other $\mathrm{gNBs}$ via the $\mathrm{X} 2-\mathrm{U}$ interface. Various combinations of eNB-5GC connections are in options 4,5 , and 7. In option 4 , the NG-RAN supports the NR-E-UTRAN Dual Connectivity (NE-DC); hence, the gNB (MN) is linked to the $5 \mathrm{GC}$ and the eNB (SN). Options 5 and 7 are two variants of LTE linked to 5GC. The architecture options 5 and 7 are known as eLTE and NGEN-DC, respectively.

The NG-RAN also supports NR-NR Dual Connectivity (NR-DC) when a gNB (MN) is linked to the 5GC and another gNB (SN). In addition, NR-DC can also be used when a UE is connected to two gNB-DUs, one serving the Master Cell Group (MCG) and the other serving the Secondary Cell Group (SCG), connected to the same gNB-CU, acting both as an MN and as an $\mathrm{SN}$.

\section{NR physical layer}

The NR physical layer [12] is in charge of uplink synchronization and timing control, multiplexing and channel coding, link adaptation, power control, random access procedures, multi-antenna mapping and processing, and beam management. It transfers information towards the higher layer (i.e. Medium Access Control or MAC) through transport channels as well as handles the mapping of transport channels to physical channels.

The physical channels defined for NR are the following.

- Physical Broadcast Channel (PBCH) carries system information for device access to the network.
- Physical Downlink Control Channel (PDCCH) is used for control information, such as scheduling decisions.

- Physical Downlink Shared Channel (PDSCH) is the main channel for unicast data transmissions regarding paging, random access, and system information.

- Physical Uplink Control Channel (PUCCH) carries information about hybrid-ARQ, received transport blocks, channel state reports, and radio resource requests.

- Physical Uplink Shared Channel (PUSCH) is the counterpart to the PDSCH.

- Physical Random Access Channel (PRACH) is used for random access.

The system operates from below 1 to $52.6 \mathrm{GHz}$ in frequency ranges FR1 and FR2 [20]. The choice of a frequency range depends on the base station deployment. At lower frequencies, wider coverage areas are available (i.e., macro-cells), whereas coverage is more limited at higher frequencies (i.e., micro- and pico-cells). The licensed spectrum provides higher quality and reliability. In contrast, the unlicensed spectrum complements by offering higher data rates and improved capacity. The main NR physical-layer components [12] are waveforms and scalable numerology, modulation schemes, frame structure, multi-antenna transmission and beamforming, and reference signals, which are described in the following subsections.

\subsection{Waveform and scalable numerology}

The 5G NR physical layer supports the Orthogonal Frequency Division Multiplexing with cyclic prefix (CPOFDM) in the downlink and CP-OFDM and Discrete Fourier Transform-spread-OFDM (DFT-s-OFDM) with CP in the uplink. The scalable OFDM numerology introduced by NR specifies SCS, Transmission Time Interval (TTI), $\mathrm{CP}$, and the number of slots. In particular, higher numerology indexes correspond to larger SCSs, ranging from 15 and $480 \mathrm{kHz}$ by following the equation:

$$
\Delta f=15 \mathrm{kHz} \times 2^{\mu} \text {, }
$$

The numerology index $\mu$ depends on various factors (i.e., service requirements, deployment type, carrier frequency, etc.). The introduction of wider SCS is essential for mitigating inter-carrier interference (ICI) and phase noise at mm-Wave frequencies. As SCS widens, the TTI assumes smaller values ranging from $1 \mathrm{~ms}$ to $31.25 \mu \mathrm{s}$. Other parameters also change from numerology to numerology. Further details are provided in Table 3.

Bandwidth is managed in terms of physical resource blocks (PRBs), each consisting of 12 subcarriers with a certain SCS. The number of PRBs depends on the channel 
Table 3 Scalable numerology. [12]

\begin{tabular}{lllll}
\hline$\mu$ & $\Delta f[\mathrm{KHz}]$ & TTI $[\mu \mathrm{s}]$ & No. symbols per slot & Cyclic prefix duration $[\mu \mathrm{s}]$ \\
\hline 0 & 15 & 1000 & 7,14 & 4.69 \\
1 & 30 & 500 & 7,14 & 2.34 \\
2 & 60 & 250 & 7,14 & 1.17 \\
3 & 120 & 125 & $2,4,7,14$ & 0.58 \\
4 & 240 & 62.5 & $2,4,7,14$ & 0.29 \\
5 & 480 & 31.25 & $2,4,7,14$ & 0.14 \\
\hline
\end{tabular}

bandwidth and SCS that are specified for FR1 and FR2 [20].

Downlink and uplink transmissions occur when at least one PRB is assigned to a UE. The radio resource allocation is managed by the gNB after performing the channel sounding that acquires the knowledge of the radio channel characteristics. Each UE sends its radio channel information to the gNB in the Channel State Information (CSI) [21] feedback. The CSI consists of the Channel Quality Indicator (CQI), precoding matrix indicator (PMI), CSI-reference signal (RS) resource indicator (CRI), SS/PBCH Block Resource indicator (SSBRI), layer indicator (LI), rank indicator (RI), and/or L1-RSRP.

In particular, the CQI is associated with the maximum supported Modulation and Coding Scheme (MCS). $R_{\min , P R B}$ is the minimum data rate per PRBs expressed in kbps, which is computed as:

$R_{\text {min }, P R B}=\frac{C R}{1024} \times M_{\text {order }} \times N_{\text {symb }} \times N_{\text {subcarrier }}$,

where $C R$ is the code rate $\mathrm{x} 1024, M_{\text {order }}$ is the modulation order (i.e., 2 for QPSK, 4 for 16-QAM, 6 for 64-QAM, and 8 for 256-QAM), $N_{\text {symb }}$ is the number of symbols that can assume values ranging from 2 to 14 (see Table 3), and $N_{\text {subcarrier }}=12$ is the number of subcarriers in a PRB.

Finally, $B_{\mu}$ is the number of bits carried by a single PRB, which can be computed as:

$B_{\mu}[$ bit $]=\frac{R_{\min , P R B, b p s}}{N_{\text {slot }, s}}=\frac{R_{\min , P R B} \times 10^{3}}{10^{3} \times 2^{\mu}}$,

where $R_{\text {min, } P R B, b p s}$ is the minimum data rate per PRB expressed in bps and $N_{\text {slot }, s}$ is the number of slots per second that depends on the numerology. As a consequence, $B_{\mu}$ is a function of the numerology. Hence, as the SCS widens, each PRB contains fewer useful bits and more overhead with respect to the PRBs of lower numerology. This develops robustness against the phase noise when operating at extremely high frequencies.

The introduction of scalable numerology allows the 5G NR to deliver dissimilar services while meeting associated requirements. On one side, low numerologies with narrower SCS are suitable to ensure wider cell coverage since a narrow SCS is robust to delay spread owing to its longer symbol duration. On the other side, high numerologies with a wider SCS increase the transmission rate by reducing the slot duration, thus offering lower latencies. Furthermore, a wide SCS is robust to the phase noise.

Conversely, wider carrier bandwidths cause device energy consumption. To cope with it, and different from LTE where all devices monitor all of the channel bandwidth, in NR each device monitors only a portion of the radio spectrum. When needed, the device can monitor the full bandwidth owing to the receiver-bandwidth adaptation. Receiving the full or a portion of carrier bandwidth is the reason to introduce the concept of Bandwidth Part (BWP), which is a fixed band over which transmissions employ the same numerology. In [22] this is defined as a subset of contiguous common resource blocks for a given numerology $\mu_{i}$ in bandwidth part $i$ on a given carrier. Two possible configurations of sub-band size are eligible for BWP [21]. The total bandwidth consists of several BWPs. Furthermore, a BWP correlates the numerology and the scheduling mechanism. Indeed, the gNB can modify the UE numerology by changing the BWP through the BW Adaptation. Although a UE can be configured with up to four BWPs in the downlink and also in the uplink, each UE can monitor a single active BWP to reduce power consumption and prolong battery life, which is important for IoT devices in mMTC-related use cases. Information about the active initial bandwidth part is obtained via the control resource set (CORESET) configuration from the $\mathrm{PBCH}$ in the downlink, whereas information on the activated initial uplink bandwidth part is transferred in the PDCCH.

\subsection{Modulation schemes}

The supported MCSs are QPSK, 16QAM, 64QAM, and 256QAM in the downlink and for CP-OFDM in the uplink, and $\pi / 2$-BPSK, QPSK, 16QAM, 64QAM, and 256QAM for DFT-s-OFDM with CP in the uplink.

\subsection{Frame structure}

The 5G NR frame structure supports Frequency Division Duplex (FDD) operating during transmissions in the paired spectrum, while Time Division Duplex (TDD) is used for 
the unpaired spectrum. Different from LTE where a subframe consists of two slots having 7 OFDM symbols, in 5G NR a subframe is formed by adjacent slots, each comprising 7 or 14 OFDM symbols for SCS $\leq 60 \mathrm{kHz}$ and 2, 4, 7 , or 14 OFDM symbols for SCS $\leq 120 \mathrm{kHz}$. The $3 \mathrm{GPP}$ standardized 255 symbol combinations, each corresponding to a slot format identified by an index (i.e., slot format index) [23]. Its OFDM symbols can be designated as downlink, uplink, or flexible. A downlink symbol is used only in the downlink direction, while no uplink transmission occurs in the same time period. On the contrary, an uplink symbol is exploited for the uplink without any overlapping transmission in the downlink. Flexible symbols can be adapted for transmissions in the downlink or in the uplink.

5G NR considers mini-slots to be the smallest scheduling units of 2, 4, or 7 symbols, which are especially important for URLLC. The URLLC transmissions are managed with the highest priority by puncturing the $1 \mathrm{~ms}$ subframe for a mini-slot duration. This enables dynamic scheduling. In the case of extremely low-latency services, the transmission start is not limited to a slot but can occur whenever the radio channel is not occupied by another transmission.

\subsection{Multi-antenna transmission and beamforming}

Since 5G NR extends the operating frequency range by comprising the frequency bands below $7 \mathrm{GHz}$ (i.e., FR1) and in the range from $24.25 \mathrm{GHz}$ to $52.6 \mathrm{GHz}$ (i.e., FR2), different antenna solutions and techniques need to be employed depending on the utilized spectrum. For lower frequencies, up to a moderate number of antennas can be activated (i.e., 32). In higher frequency bands, the transmission is characterized by a considerable signal attenuation that limits the network coverage. To overcome this limitation, one of the key features is the adoption of a large number of multi-antenna elements having a given aperture to increase the transmission/reception capability of multiuser MIMO (MU-MIMO) and beamforming.

Since managing transmissions in higher frequency bands is complicated, beam management is necessary to establish the correspondence between the directions of the transmitter-and the receiver-side beams by identifying the most suitable beam pair for both downlink and uplink. 5G NR offers a new type of beam management, wherein the BS sweeps the candidate radio transmitter beams sequentially in time, while the UE maintains an appropriate radio receiver beam to activate the reception of the selected radio transmitter beam. To achieve this, beam management consists of the initial beam establishment to find the first beam pair, the beam adjustment to adapt the beam pair as the device moves, and the beam recovery to handle the beam pair failure in the case of rapid environmental changes. Hence, the complete beam management functionality establishes, monitors, adjusts, and recovers the beam pair to allow for communication at higher frequencies.

However, beamforming procedures are critical concerning the spectral efficiency and reliable coverage. Therefore, an accurate design of the above mentioned procedures is essential to meet the stringent reliability requirements of URLLC.

\subsection{Reference signals}

In NR, reference signals are transmitted only if necessary; they are as follows:

- Demodulation Reference Signal (DMRS) is UE-specific and measures the radio channel for demodulation to support multiple-layer MIMO transmission and lowlatency applications.

- Phase-Tracking Reference Signal (PTRS) is UE-specific and exploited at mmWave frequencies to mitigate the phase noise causing phase rotation of subcarriers in an OFDM signal. PTRS compensates for the oscillator phase noise.

- Channel-State Information Reference Signal (CSI-RS) is received by the UE to evaluate the channel; further, the channel quality information is transferred to the gNB.

- Sounding Reference Signal (SRS) is transmitted by the UE to help the gNB in estimating the CSI for scheduling and link adaptation. SRS is also utilized for reciprocity in massive MIMO and UL beam management.

- Positioning Reference Signal (PRS) is a new reference signal that supports DL-based positioning. The UE reports the times of arrival of PRSs transmitted by multiple base stations; in such a way, the location server is able to determine the UE position.

- Primary synchronization signal (PSS) is a physical layer-specific signal that helps the UE to get radio frame boundary and to detect the cell identity (ID).

- Secondary synchronization signal (SSS) is a physical layer-specific signal that, in addition to the PSS, helps the UE to detect the cell ID group. 


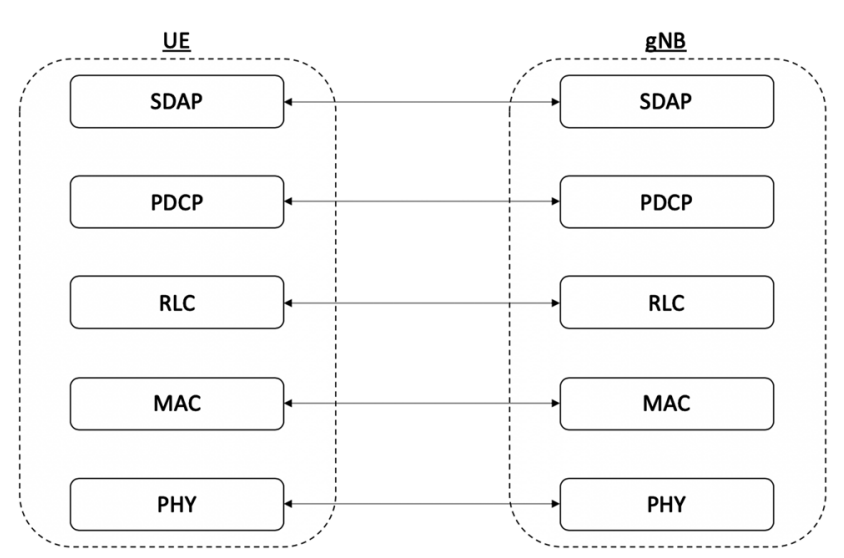

Fig. 4 User-plane protocol stack architecture

\section{User-plane protocols}

The user-plane protocols [24] facilitate the actual Packet Data Unit (PDU) session service by transporting user data through the radio access layer. Figure 4 shows the userplane protocol stack architecture.

\subsection{Medium access control (MAC)}

The MAC layer provides a connection to the Layer 2 Radio-Link Control (RLC) layer through various logical channels, each characterized by the type of the transferred information. The control logical channels are:

- Broadcast Control Channel (BCCH), which transmits system information to all the network devices in the case of standalone operation. For non-standalone operations, there is no $\mathrm{BCCH}$ because the system information is provided by the LTE system.

- Paging Control Channel (PCCH), which is used for paging the devices when their location is unknown by the network in the case of standalone operation. For non-standalone operations, there is no $\mathrm{PCCH}$ because paging is provided by the LTE system.

- Common Control Channel (CCCH), which transmits control information under random access regime.

- Dedicated Control Channel (DCCH), which is the logical channel used for transmission of the control information to and from a device.

The logical channel related to traffic is Dedicated Traffic Channel (DTCH), which is used for the data transmission to and from a device over a unicast link in the downlink or uplink, respectively. The PHY layer provides its services to the MAC layer through the transport channels. A transport channel refers to how the information is transferred over the radio interface.

The downlink transport channels are:
- Broadcast Channel (BCH), which is used for the transmission of Master Information Block (MIB) that is a part of the BCCH system information.

- Downlink Shared Channel (DL-SCH), which is the main channel for downlink data transmission in NR and supports its key features, such as dynamic rate adaptation and channel-dependent scheduling.

- Paging Channel (PCH), which carries paging information from the $\mathrm{PCCH}$ channel by saving the battery power of the devices supporting discontinuous reception (DRX).

The uplink transport channels are:

- Uplink Shared Channel (UL-SCH), which is the main channel for uplink data transmission.

- Random Access Channel (RACH), which is another transport channel even though it does not carry transport blocks.

Figure 5 depicts the mapping among physical, transport, and logical channels in both uplink and downlink directions.

The MAC layer is responsible for multiplexing/demultiplexing, for managing different numerologies and scheduling, for hybrid-ARQ retransmissions, and for handling the priority between the UEs via dynamic scheduling. Extended MAC functionality is introduced by 5G NR: e.g., the MAC PDU is changed. MAC elements used for control signaling are at the beginning of the MAC PDU in the downlink and the end of the MAC PDU in the uplink. Hence, the NR MAC layer header structure is more efficient in supporting low-latency requirements.

The procedure for creating MAC PDUs is known as Logical Channel Prioritization (LCP). In NR, there is also a need for LCP enhancement due to scalable numerology and TTIs, each suited for a specific service type. The LCP handles the mapping of a logical channel onto certain numerology/TTI and includes URLLC traffic prioritization. Owing to the latter, URLLC applications are delivered as soon as they are requested, thus allowing to meet the reliability and very low latency requirements of URLLC services. Hence, LCP aims to let the MAC entity learn the numerology/TTI from the PHY layer.

Beyond improved PDU format, the NR MAC layer adopts new scheduling request (SR) functionality. The SR is utilized to request the UL-SCH resources to establish a data transmission. Different from LTE SR, where a UE supports only one SR resource, the NR MAC entity is configured such that a UE may support zero, one, or more SR configurations, each consisting of multiple PUCCH resources for different BWPs and corresponding to one or more logical channels. A logical channel is configured to support only one PUCCH resource for SR per BWP. 
Fig. 5 Channels mapping

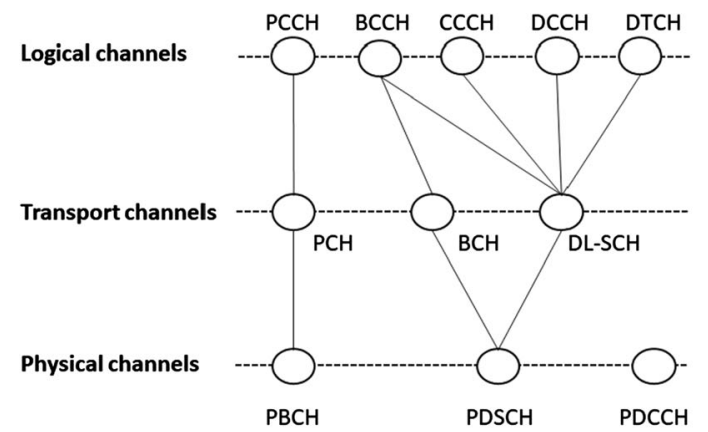

Downlink

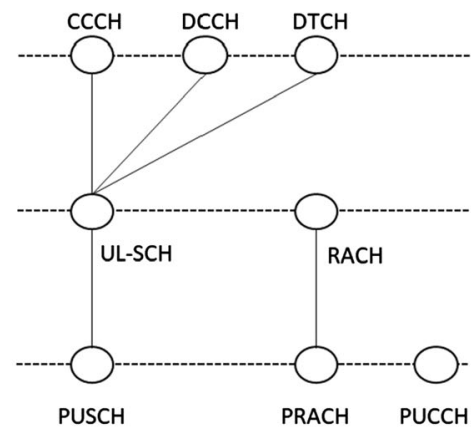

Uplink
The SR configuration/resource of the logical channel that has triggered a Buffer Status reporting (BSR), which provides information about uplink data volume in the MAC entity to the serving $\mathrm{gNB}$, is considered as the corresponding SR configuration/resource for the triggered SR. Each logical channel may be allocated to a Logical Channel Group (LCG), while the maximum number of LCGs is increased to eight for NR as opposed to 4 LCGs supported by LTE.

\subsection{Radio link control (RLC)}

The RLC layer handles data transfer and is mainly based on the LTE RLC. It offers three transmission options: (i) Transparent Mode (TM), (ii) Unacknowledged Mode (UM), and (iii) Acknowledged Mode (AM). RLC also performs segmentation by considering the sequence number and the segment offset, transfers upper-layer PDUs, corrects errors via Automatic Repeat Request (ARQ), and detects protocol errors.

Different from LTE RLC, NR RLC does not order the Service Data Units (SDUs) in sequence to reduce the overall latency; hence, packets can be forwarded towards the upper layers without waiting for re-transmissions of previously missing packets. Furthermore, to meet the NR latency requirements extremely important for URLLC applications, the RLC does not support concatenation (now introduced at the MAC layer), differently from LTE. As a result, NR RLC PDUs can be assembled before receiving the scheduling decision. Once the latter is made, the PDUs are transferred to the MAC layer immediately. On the other hand, LTE RLC PDUs are assembled and then forwarded to the lower layer.

\subsection{Packet data convergence protocol (PDCP)}

The PDCP layer is in charge of header compression and decompression, user data transfer, handling re- transmissions, reordering, and detection of duplicates during handover. The re-ordering functionality is moved from RLC to PDCP in NR. The PDCP layer is also in charge of ciphering, deciphering, and integrity protection, as well as duplication, which is a new PDCP function. Accordingly, user packets are re-transmitted to the gNB several times such that at least one copy is received correctly. If more than a single copy of the same PDU is received, the PDCP removes any duplicates. PDCP duplication is also used (i) in case of transmission via multiple cells to meet highreliability requirements of URLLC and (ii) in dual-connectivity scenarios.

\subsection{Service data adaptation protocol (SDAP)}

The challenge of meeting various QoS requirements of dissimilar services and use cases has already been addressed by LTE. However, QoS handling in NR needs to be improved under network slicing. Hence, a new userplane protocol layer, namely, Service Data Adaptation Protocol (SDAP), was defined to manage QoS when connected to the 5GC. Each PDU session thus consists of QoS flows and data radio bearers. The IP packets are associated with the QoS flows according to their requirements, and are labeled with an identifier, i.e., QoS Flow Identifier (QFI). Hence, SDAP provides the mapping of a QoS flow from the $5 \mathrm{GC}$ and a data radio bearer, while marking the QFI.

The correspondence between QoS flows and data radio bearers in the uplink is as follows. Explicit mapping considers the configuration from the QoS flow to the data radio bearer in the device through RRC signaling. Alternatively, reflective mapping is a novel NR functionality when connected to the 5GC. Here, a device observes the QFI in the downlink by inferring which IP packets are associated with a QoS flow and a data radio bearer. 
Fig. 6 Control-plane protocol stack architecture

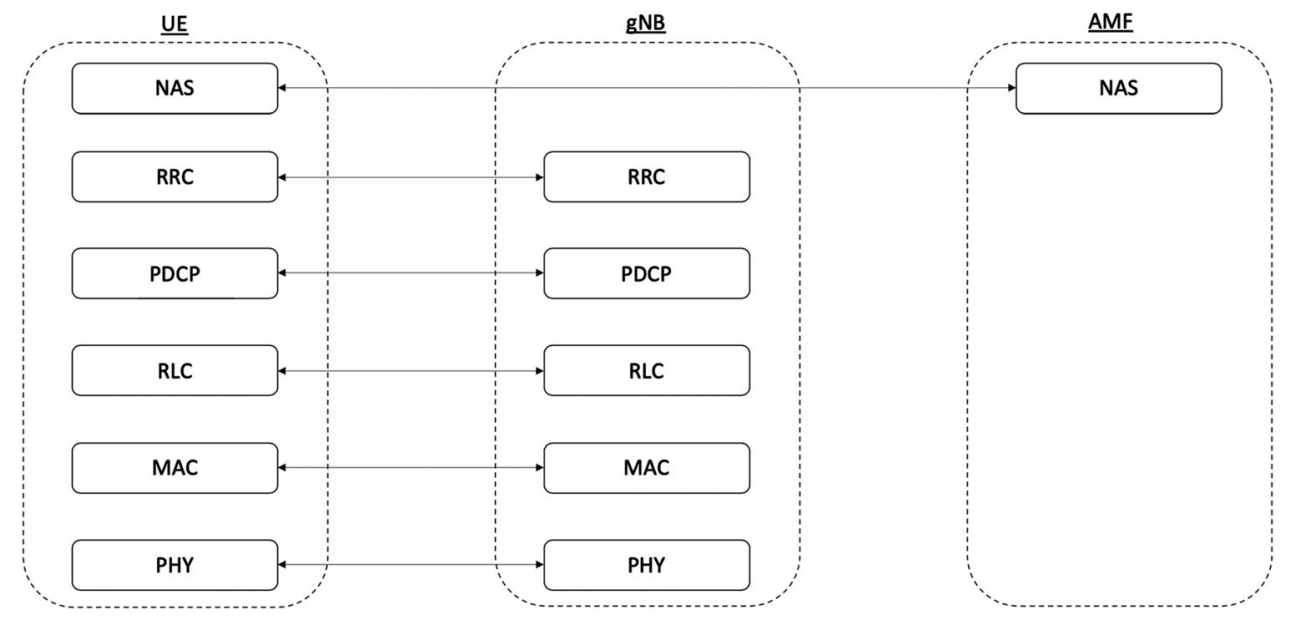

\section{Control-plane protocols}

The control-plane protocols [24] establish PDU sessions and manage connections between the UE and the network mindful of service requests, transmission resources, and handover occurrences.

Figure 6 shows the control-plane protocol stack architecture.

The control-plane stack has the same protocol layersPHY, MAC, RLC, and PDCP-as described in Sect. 4.

The protocol layer having solely control-plane functionality is Radio Resource Control (RRC), which operates between a device and its gNB. It handles the paging procedure initiated by 5GC or NG-RAN. RRC also establishes, maintains, and releases the RRC connection between a UE and NG-RAN by including security functions (i.e., key management), mobility functions (i.e., handover, UE cell selection and reselection, inter-RAT mobility), and QoS management functions. The RRC also helps detect and recover from radio link failures. Each NR device supports three RRC states, two of which-IDLE and CONNECTED-are the same as in LTE. Moreover, NR introduces INACTIVE as an intermediate state between IDLE and CONNECTED to reduce the energy consumption and prolong the battery life of IoT and mMTC-related devices. Further information on RRC state transitions can be found in [24]. With NR, the transmission of messages between RRC and NAS layer occurs owing to Signaling Radio Bearers (SRBs), which are:

- SRB0: exploits the CCCH logical channel for RRC messages;

- SRB1: exploits the DCCH logical channel for both RRC and NAS messages before establishing SRB2;

- SRB2: exploits the DCCH logical channel for NAS messages and has a lower priority than SRB1;
- SRB3: exploits the DCCH logical channel for particular RRC messages when UE operates in dual-connectivity mode.

The SRB1 and SRB2 are also used in NR for integrity protection and ciphering as well as support the split of SRB for all Multi-Radio Dual Connectivity (MR-DC) options. The latter is not available for SRB0 and SRB3. Further, the SRB split and SRB3 are the two new features introduced by NR on top of LTE.

\section{Performance evaluation of 5G NR}

The below simulation results were obtained with our MATLAB tool that follows 3GPP guidelines on scenarios and requirements for the 5G NR technology [25]. The main simulation parameters are listed in Table 4.

In this work, we characterize the performance in terms of peak data rate and peak spectral efficiency for different numerologies (i.e., $\mu=0,1,2,3$ ) and under varying channel bandwidth ${ }^{1}$ when delivering eMBB services in a $5 \mathrm{G}$ NR system. For the sake of completeness, we also evaluate the performance in terms of energy consumption and reliability for specified numerologies under varying CQIs when performing mMTC and URLLC, respectively. The selected parameters were evaluated by considering the transmission bandwidth configuration for both FR1 and FR2 [20]. Further, we computed the number of useful bits per PRB for each numerology by considering the CQIMSC mapping parameters in [21]. The motivation behind this performance analysis is to understand how scalable numerology affects the volume of data carried by a single PRB.

${ }^{1}$ It is worth noting that in 3GPP Release 16 of NR [20], the number of PRBs is specified for numerologies from 0 to 3 , whereas the number of PRBs for numerologies 4 and 5 is not available in that release. 
Table 4 Simulation Parameters

\begin{tabular}{ll}
\hline Parameter & Value \\
\hline Cell layout & Hexagonal grid \\
Inter-site distance & $500 \mathrm{~m}$ \\
Scheduling frame & $10 \mathrm{~ms}$ \\
$\mu$ & $0,1,2,3$ \\
TTI & $1 \mathrm{~ms}, 0.5 \mathrm{~ms}, 0.25 \mathrm{~ms}, 0.125 \mathrm{~ms}$ \\
RB size & $12 \mathrm{sub}-\mathrm{carriers}$ \\
Sub-carrier spacing & $15 \mathrm{kHz}, 30 \mathrm{kHz}, 60 \mathrm{kHz}, 120 \mathrm{KHz}$ \\
Component carrier & 1 \\
Modulation order & $8(256-\mathrm{QAM})$ \\
CQI & 15 \\
Tx direction & Downlink \\
Number of OFDM symbols & 14 \\
Number of UE & 100 \\
\hline
\end{tabular}

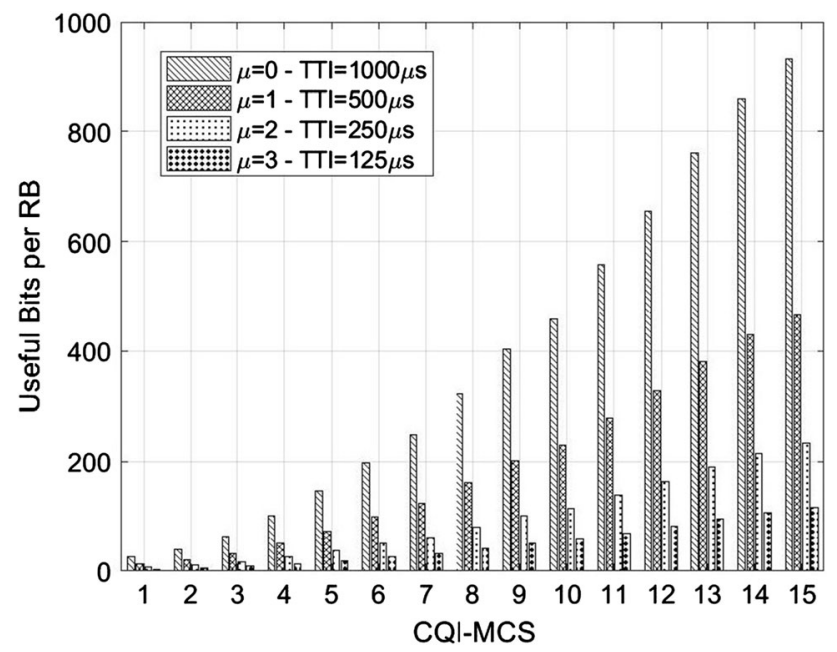

Fig. 7 Useful bits per PRB under varying CQI

From this perspective, Fig. 7 indicates the number of useful bits per PRB (computed as in Eq. 3) for different TTI durations according to the selected numerology when varying the UE channel conditions. As expected, in all cases, the parameter of interest increases with the CQI level, whereas the number of useful bits decreases, for a given CQI, when considering higher numerologies.

Reducing the end-to-end latency is possible by operating at higher frequencies and under high numerologies, wherein OFDM symbol duration is shorter and, consequently, the SCS is wider. Higher frequency also displays more pronounced path-loss degradation: as the signal is attenuated and distorted during its propagation at higher frequencies, wider SCS is required to compensate for the observed effect. Conversely, the peak data rate per TTI decreases for high numerologies when considering fixed

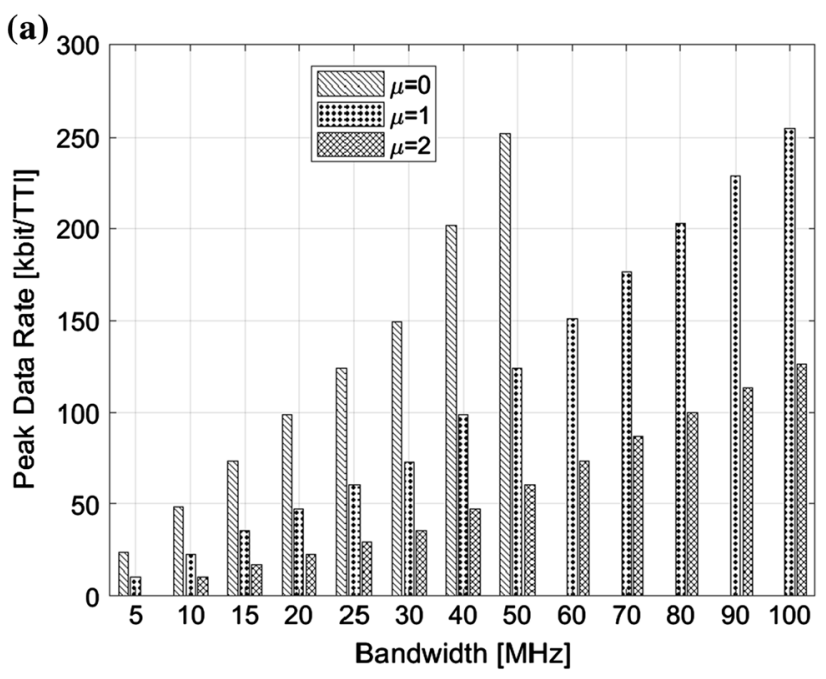

(b)

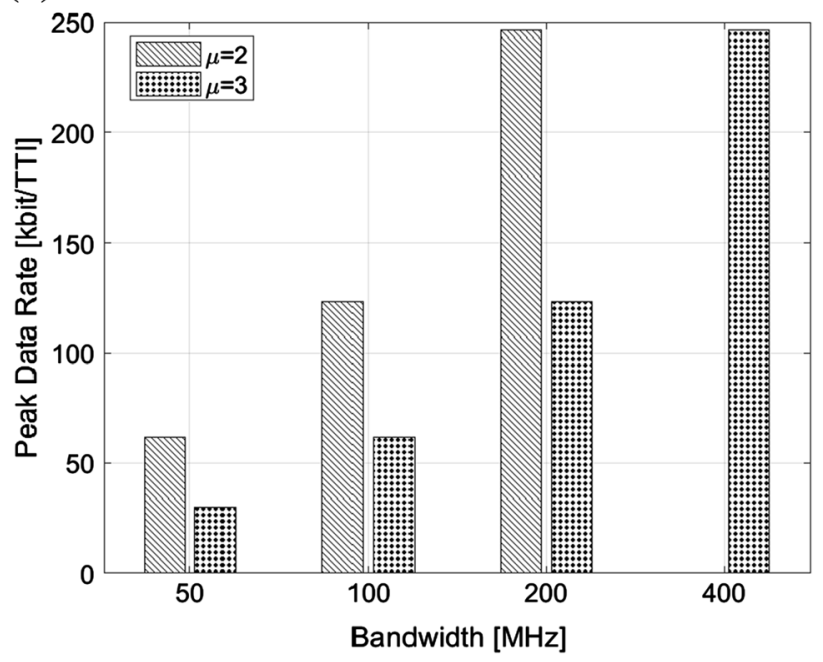

Fig. 8 Peak data rate for a FR1 and b FR2

bandwidth, as shown in Fig. 8. This is because the number of PRBs per TTI becomes lower due to wider SCS with higher numerology. Under increasing channel bandwidth, the peak data rate follows a growing trend due to more available PRBs.

When considering higher numerologies, a consequence of the peak data rate decrease is the reduction in terms of peak spectral efficiency in a TTI, as shown in Fig. 9. Actually, considering higher frequencies leads to more pronounced path-loss. Since the signal is attenuated and distorted during the propagation on higher frequencies, more overhead bits are needed to compensate for these effects. This means that the radio spectrum is not fully exploited for the delivery of useful data, hence, a reduction in the system spectral efficiency is shown.

Figure 10 indicates the energy consumption required for receiving a certain number of bits per $\mathrm{RB}$ depending on both CQI and numerology. It can be noticed that the curve 

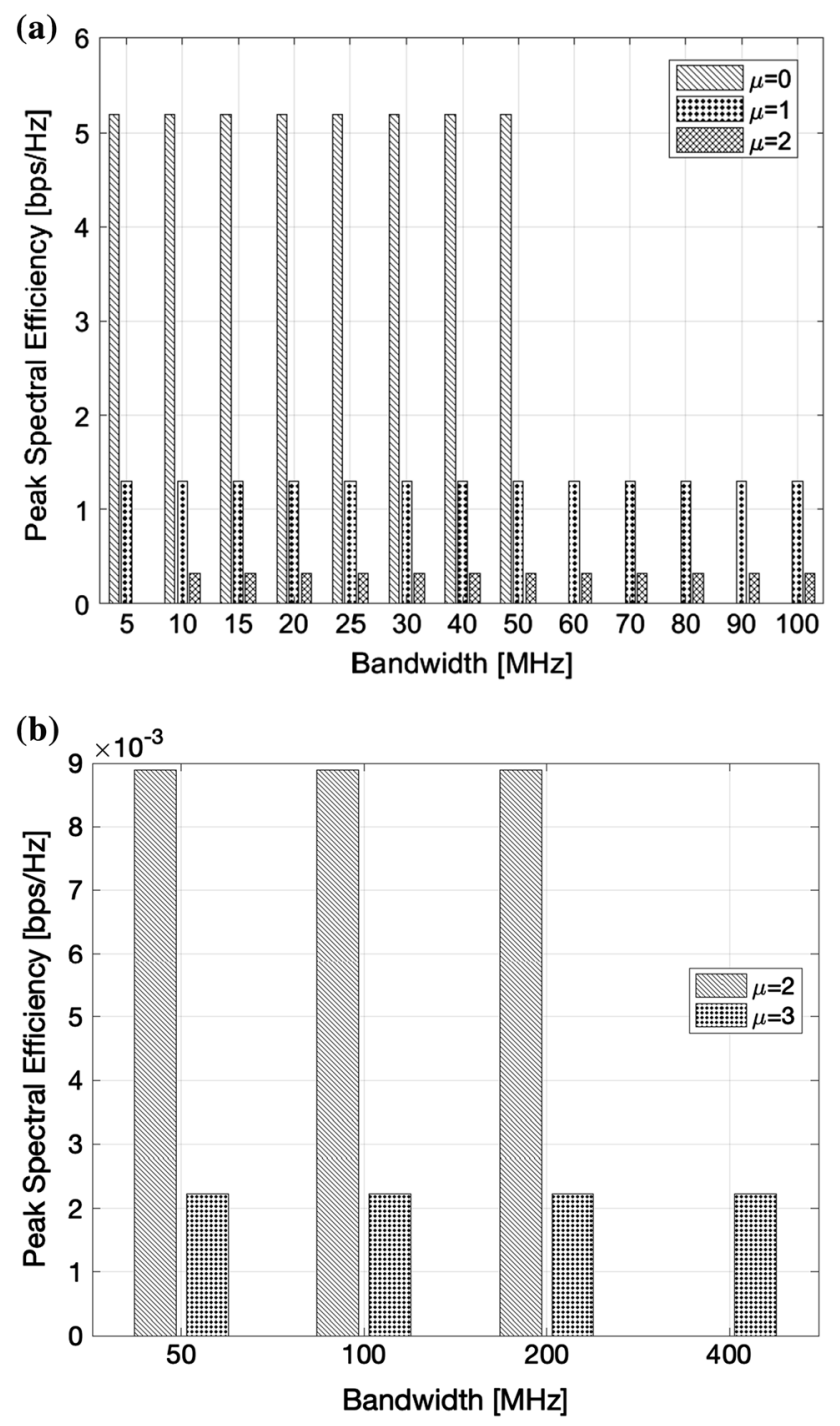

Fig. 9 Peak spectral efficiency for a FR1 and b FR2

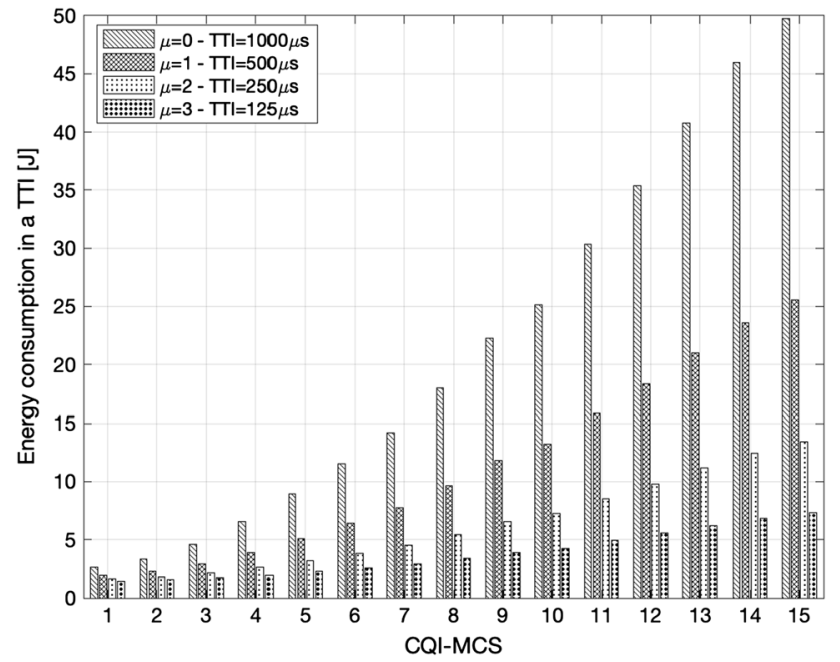

Fig. 10 Energy consumption in a TTI under varying CQI follows a growing trend for all numerologies. This, indeed, depends on the number of transmitted/received bits, which increases when adopting less robust modulation schemes (i.e., higher MCS). On the other hand, for even higher numerologies, the number of transmitted/received bits is lower, and consequently, the energy consumed by devices decreases. Therefore, for mMTC, the choice of the appropriate numerology can be made according to the requirements of energy consumption and battery life.

Figure 11 provides a measure of the reliability intended, according to [25], as the success probability of transmitting a data packet within a certain delay. Generally, the reliability requirement is dependent of deployment and operation scenario. We show in Fig. 11 the reliability [\%] for a general URLLC traffic by considering a 32 bytes packet [25]. We simulated that the transmission occurs in a TTI depending on the numerology as shown in Table 3. The reliability for the correct reception of the transmitted data grows when increasing the CQI and decreases when considering even higher numerology. However, it is worth noting that we considered the slot duration specified by each numerology and only one PRB. Therefore, to achieve $100 \%$ of reliability with all values of CQI and numerology, 14 PRBs are needed in a mini-slot lasting $0.125 \mathrm{~ms}$ (i.e., $\mu=2$ ). This allows to satisfy the reliability and low-latency requirements of URLLC and does not give rise to concern since NR extends the radio spectrum; indeed, with the FR2 a channel bandwidth up to $400 \mathrm{MHz}$ (i.e., 264 PRBs) is possible.

By analyzing the obtained results, we establish that: (i) lower numerologies are better suited for eMBB services with their higher volumes of data and without the stringent requirements in terms of latency; (ii) higher numerologies satisfy the requirement of (ultra-)low latency, which is essential for URLLC, at the expense of some reduction in

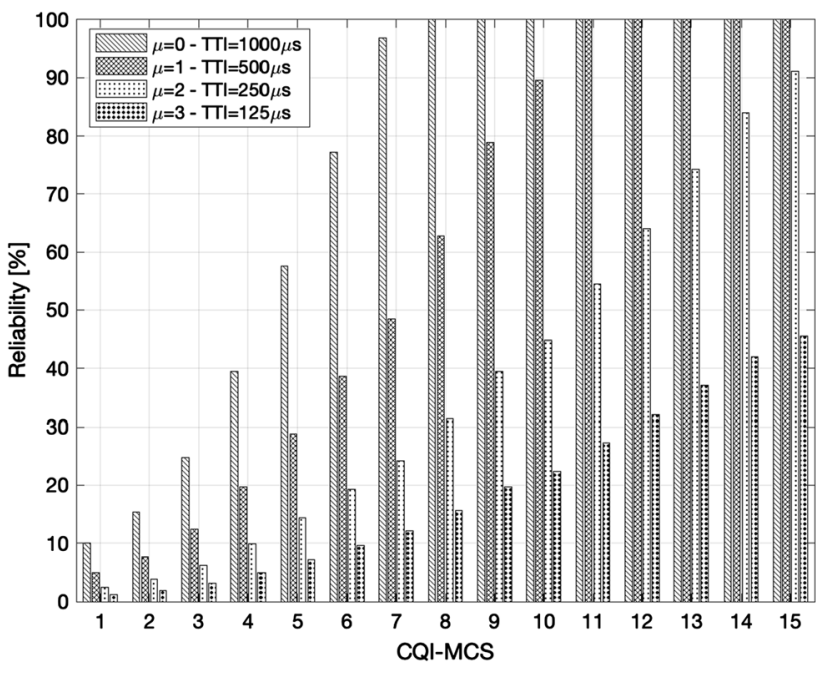

Fig. 11 Reliability under varying CQI 
the peak data rate; (iii) higher numerologies can also be exploited at higher frequencies while lower numerologies suit lower frequencies since the number of useful bits per PRB delivered in a TTI decreases under higher numerologies. As an example, a typical URLLC transmission comprising of few bits (e.g., data exchange between sensors of vehicles for collision avoidance), may occur at higher frequencies, which allows satisfying latency requirements but demands a wider SCS to mitigate the phase noise effect.

\section{Open challenges and future research directions}

The 5G NR technology revolutionizes the previous radio technology while maintaining compatibility with LTE and is designed forward compatible with future technologies. The main NR features have been thought to support several types of services with different requirements, such as extreme data rates, elevated reliability, and very low latency.

The global market becomes increasingly demanding and is directed towards new use cases that will characterize the future radio technology. In [26], the ITU identifies the following main categories: Holographic Type Communications (HTC) to holographically reproduce subjects in remote places; Multi-Sense Networks to live fully immersive experiences; Time Engineered Applications to allow sensors to autonomously and quickly react in front of unpredictable events without human intervention; and Critical Infrastructure to ensure safety anywhere and anytime. In this regard, one of the main challenges is to adapt NR as much as possible in order to be able to meet the more stringent requirements of new applications unthinkable until now.

The mainstream specification work on Release 17 is ongoing. There are multiple areas to be considered and, realistically, only a subset of them (about 20-30\%) can be addressed. On the one hand, many items are completely new, whereas several of those have already been specified in earlier releases and are to be expanded in Release 17. The more innovative topics are the following:

- NR Light: improved operation of mid-tier NR devices (e.g., higher-end MTC devices like security cameras, wearables, etc.), which includes the power saving aspects. Indeed, among the new use cases that will be considered, there is the support of devices with reduced capabilities for IoT or MTC use cases requiring fast data speeds but being also less expensive than the $5 \mathrm{G}$ NR devices deployed today.
- NR above $52.6 \mathrm{GHz}$ : possibility to extend the frequency bands up to $114 \mathrm{GHz}$ by studying suitable waveforms. The spectrum falling in the range between $130 \mathrm{GHz}$ and $300 \mathrm{GHz}$ is known as sub- $\mathrm{THz}$ and between $300 \mathrm{GHz}$ and $3 \mathrm{THz}$ as $\mathrm{THz}$ band. $\mathrm{THz}$ communications pave the way to $6 \mathrm{G}$ owing to the capability to achieve elevated datarates and extreme capacity in short distance [27]. However, THz communications suffer from high signal attenuation due to path loss and molecular absorption thus needing innovative solutions, such as Intelligent Reflecting Surfaces (IRS) [28].

- Multi-SIM operation: specification enhancements, especially in the area of paging coordination, for the more efficient and predictable operation of high numbers of multi-SIM devices. Due to the current lack of unified solutions for multi-SIM operation, several critical issues must be addressed such as ongoing service interruption, unwanted resource wastage, misleading assumption of reachability, and collision of paging occasions.

- NR multicast broadcast: support for multicast transmissions by focusing on single-cell multicast functionality with the evolution towards multi-cell. Mechanisms for the multi-cell coordination are essential to support Single Frequency Network (SFN) transmissions. Furthermore, modification to the NR protocol stack are required to enable the NR mixed mode (i.e., the coexistence of unicast and multicast) [29].

- NR for Non-Terrestrial Networks: defining the requirements as well as the candidate layer 1 solutions and their implications. NR protocols need to be adapted to: compensate for the Doppler effect due to the satellite motion, manage the long propagation delay, handle the fast sweep of satellite footprints across the ground, and improve the link budget.

Furthermore, new approaches for efficient radio resource management are needed to overcome the issue of internumerology interference when dissimilar services are demanded and multiple numerologies are supported in the NR system. Finally, innovative techniques of puncturing are essential to handle different traffic request by giving priority to URLLC services.

\section{Conclusions}

In this article, we surveyed the main 5G NR system design features with the topics covering the key innovative aspects introduced in the system architecture and protocol stack (i.e., physical layer, user-/control-plane protocols). This information is representative of the latest Releases 16 of the 38th series of 3GPP specifications. Furthermore, we 
assessed the system performance by analyzing the results of system-level simulations. Based on these results, we projected useful considerations regarding the impact of scalable numerology on the 5G NR performance. Finally, open challenges and future research directions were briefly outlined.

Funding Open access funding provided by Università degli Studi Mediterranea di Reggio Calabria within the CRUI-CARE Agreement.

Open Access This article is licensed under a Creative Commons Attribution 4.0 International License, which permits use, sharing, adaptation, distribution and reproduction in any medium or format, as long as you give appropriate credit to the original author(s) and the source, provide a link to the Creative Commons licence, and indicate if changes were made. The images or other third party material in this article are included in the article's Creative Commons licence, unless indicated otherwise in a credit line to the material. If material is not included in the article's Creative Commons licence and your intended use is not permitted by statutory regulation or exceeds the permitted use, you will need to obtain permission directly from the copyright holder. To view a copy of this licence, visit http://creativecommons. org/licenses/by/4.0/.

\section{References}

1. Bhushan, N., Ji, T., Koymen, O., Smee, J., Soriaga, J., Subramanian, S., \& Wei, Y. (2017). Industry perspectives: 5G air interface system design principles. IEEE Wireless Communications, 24(5), 6-8.

2. ITU-R (2017). Minimum requirements related to technical performance for IMT2020 radio interface(s). Report ITU-R M.24100 .

3. Parkvall, S., Dahlman, E., Furuskär, A., \& Frenne, M. (2017). 5G NR: the next generation wireless access technology. IEEE Communications Standards Magazine, 1(4), 24-30.

4. Zaidi, A., Baldemair, R., Tullberg, H., Björkegren, H., Sundström, L., Medbo, J., et al. (2016). Waveform and numerology to support 5G services and requirements. IEEE Communications Magazine, 54(11), 90-98.

5. 3GPP (2020). TS 36.211; Evolved Universal Terrestrial Radio Access (E-UTRA); Physical channels and modulation; Rel. 16. 3GPP: Sophia Antipolis Valbonne, France.

6. Liu, J., Au, K., Maaref, A., Luo, J., Baligh, H., Tong, H., et al. (2018). Initial access, mobility, and user-centric multi-beam operation in 5G new radio. IEEE Communications Magazine, 56(3), 35-41.

7. Biyabani, S. R., Khan, R., Alam, M. M., Biyabani, A. A., \& McCune, E. (2019). Energy efficiency evaluation of linear transmitters for 5G NR wireless waveforms. IEEE Transactions on Green Communications and Networking, 3(2), 446-454.

8. Ramos, E., \& Orsino, A. (2018). Effects of beamforming and antenna configurations on mobility in 5G NR. Ericsson Research, https://doi.org/10.13140/RG.2.2.22822.98887.

9. Rinaldi, F., Määttänen, H.-L., Torsner, J., Pizzi, S., Andreev, S., Iera, A., et al. (2021). Broadcasting services over 5G NR enabled multi-beam non-terrestrial networks. IEEE Transactions on Broadcasting, 67(1), 33-45.

10. Vamvakas, P., Tsiropoulou, E. E., \& Papavassiliou, S. (2019). Dynamic spectrum management in $5 \mathrm{G}$ wireless networks: a real- life modeling approach. IEEE INFOCOM 2019 - IEEE Conference on Computer Communications, pp. 2134-2142.

11. Makki, B., Chitti, K., Behravan, A., \& Alouini, M.-S. (2020). A survey of NOMA: current status and open research challenges. IEEE Open Journal of the Communications Society, 1, 179-189.

12. Zaidi, A., Baldemair, R., Faxér, S., Molés-Cases, V., \& Wang, Z. (2017). Designing for the future: the 5G NR Physical Layer. Ericsson Technology Review.

13. Lin, X., Li, J., Baldemair, R., Cheng, T., Parkvall, S., Larsson, D., et al. (2019). 5G new radio: unveiling the essentials of the next generation wireless access technology. IEEE Communications Standards Magazine, 3(3), 30-37.

14. Sanfilippo, G., Galinina,O., Andreev, S., Pizzi, S., \& Araniti, G. (2018). A concise review of $5 G$ new radio capabilities for directional access at mmWave frequencies. Internet of Things, smart spaces, and next generation networks and systems, pp. 340-354. Springer.

15. 3GPP (2020). TS 38.300; NR; NR and NG-RAN overall description, Stage 2; Rel. 16. 3GPP: Sophia Antipolis Valbonne, France.

16. 3GPP (2021). TS 38.401; NG-RAN; Architecture description; Rel. 16. 3GPP: Sophia Antipolis Valbonne, France.

17. 3GPP (2020). TS 37.340; Evolved Universal Terrestrial Radio Access (E-UTRA) and NR; Multi-connectivity; Stage 2; Rel. 16. 3GPP: Sophia Antipolis Valbonne, France.

18. 3GPP (2020). TS 23.501; System architecture for the 5G system; Rel. 16. 3GPP: Sophia Antipolis Valbonne, France.

19. 3GPP (2014). TR 36.842; Study on small cell enhancements for E-UTRA and E-UTRAN; Higher layer aspects; Rel.12. 3GPP: Sophia Antipolis Valbonne, France.

20. 3GPP (2020). TS 38.104; NR; Base Station (BS) radio transmission and reception; Rel. 17. 3GPP: Sophia Antipolis Valbonne, France.

21. 3GPP (2020). TS 38.214; NR; Physical layer procedures for data; Rel. 16. 3GPP: Sophia Antipolis Valbonne, France.

22. 3GPP (2020). TS 38.211; NR; Physical channels and modulation; Rel. 16. 3GPP: Sophia Antipolis Valbonne, France.

23. 3GPP (2020). TS 38.213; NR; Physical layer procedures for control; Rel.16; 3GPP: Sophia Antipolis Valbonne, France.

24. 3GPP (2017). TR 38.804; Study on new radio access technology; Radio interface protocol aspects; Rel.14. 3GPP: Sophia Antipolis Valbonne, France.

25. 3GPP (2020). TR 38.913; Study on scenarios and requirements for next generation access technologies; Rel.16. 3GPP: Sophia Antipolis Valbonne, France.

26. FG-NET-2030 (2019). Network 2030: A blueprint of technology, applications and market drivers towards the Year 2030 and Beyond. White paper.

27. Polese, M., Jornet, J., Melodia, T., \& Zorzi, M. (2020). Toward end-to-end, full-stack 6G Terahertz networks. IEEE Communications Magazine, 58(11).

28. Gong, S., Lu, X., Hoang, D. T., Niyato, D., Shu, L., Kim, D. I., \& Liang, Y. C. (2020). Toward smart wireless communications via intelligent reflecting surfaces: a contemporary survey. IEEE Communication Surveys \& Tutorials, 22(4), 2283-2314.

29. Garro, E., Fuentes, M., Carcel, J. L., Chen, H., Mi, D., Tesema, F., et al. (2020). 5G mixed mode: NR multicast-broadcast services. IEEE Transactions on Broadcasting, 66(2), 390-403.

Publisher's Note Springer Nature remains neutral with regard to jurisdictional claims in published maps and institutional affiliations. 


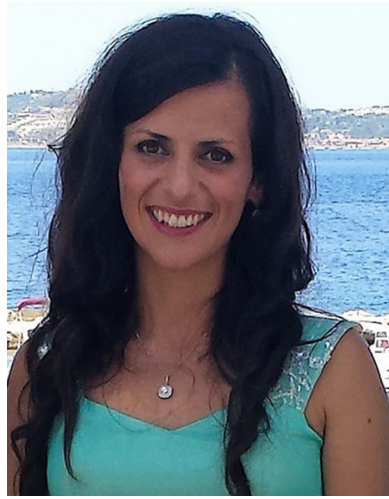

Federica Rinaldi is a Research Assistant at University Mediterranea of Reggio Calabria, Italy, where she received the Ph.D. in Information Engineering in 2021. Her current research interests include NonTerrestrial Networks (NTN), integrated terrestrial-NTN systems, RRM for multicast/ broadcast service delivery, and D2D in 5G/B5G networks.

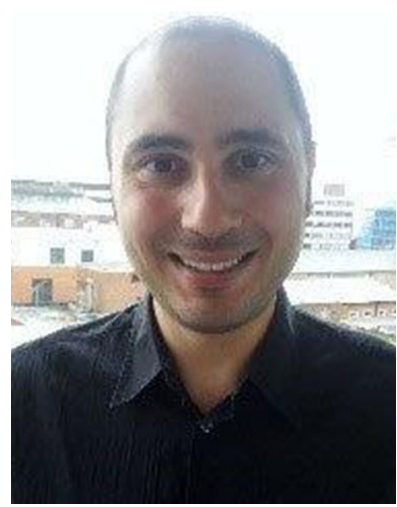

Alessandro Raschellà received the B.Sc. and M.Sc. degrees in Telecommunications Engineering from the University Mediterranea of Reggio Calabria (UNIRC), Italy in 2004 and 2007, respectively, and the $\mathrm{Ph} . \mathrm{D}$. degree in Wireless Communications from the Universitat Politècnica de Catalunya (UPC), Barcelona, Spain in 2015. From 2007 to 2009, he was a research assistant with UNIRC. He joined the School of Computer Science and Mathematics of Liverpool John Moores University (LJMU), UK in 2015, working as a Research Fellow and then, from 2019 as a Lecturer. His research interests include wireless networks optimization, softwaredefined networking, cognitive radio and heterogeneous networks.

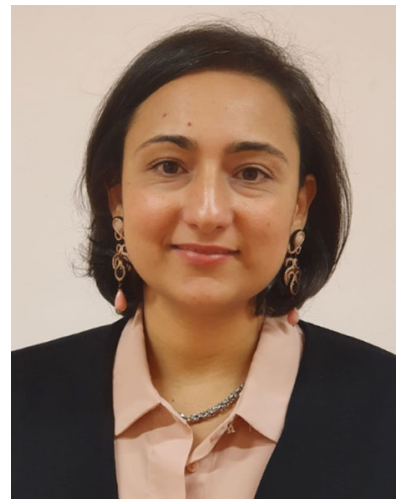

Sara Pizzi is an assistant professor in Telecommunications at University Mediterranea of Reggio Calabria, Italy, where she received the Ph.D. degree (2009) in Computer, Biomedical and Telecommunication Engineering. Her current research interests focus on NTN, RRM for multicast service delivery, D2D and MTC over 5G/B5G networks. 